Dhaka Univ. J. Biol. Sci. 24(1): 65-72, 2015 (January)

\title{
QUANTATIVE ASSESMENT OF KATO-KATZ THICK SMEAR AND FORMOL-ETHER CONCENTRATION TECHNIQUE FOR THE DIAGNOSIS OF ASCARIS LUMBRICOIDES
}

\author{
Hamida Khanum* ${ }^{*}$ Mafruha Alam, Imtiaz Khalil and Rashidul Haque ${ }^{1}$ \\ Parasitology Laboratory, Department of Zoology, University of Dhaka, Dhaka-1000, Bangladesh
}

Key words: Kato-katz, Concentration technique, Ascaris lumbricoides

\begin{abstract}
The quantitative analysis of A. lumbricoides eggs in stool of 205 subjects (male 101 and female 104) showed that the mean eggs per gram(EPG) by KatoKatz $(K K)(E P G=11130.89)$ was markedly higher than those of Formal-Ether concentration technique $(\mathrm{FE})(\mathrm{EPG}=1593.32)$. Co-efficient of correlation was significant $\left(\mathrm{r}=0.453^{* *}\right)$ at $\mathrm{p}=0.01$ level. Age based comparison among the subjects also revealed that $\mathrm{KK}$ had a better quantification of $A$. lumbricoides eggs than FE.
\end{abstract}

\section{Introduction}

Intestinal parasitic nematode Ascaris lumbricoides, that causes ascariasis, an infection that has a cosmopolitan distribution and is highly prevalent in warm tropical countries ${ }^{1-}$ 3). Ascariasis is often manifested by protein-energy malnutrition, losing of appetite and vitamin-A mal-absorption ${ }^{(4)}$. In hyper-infective children, worm masses can cause obstruction or perforation of the intestine and occasionally obstruction of the bile ducts and pancreatic ducts(5). Heavy infections are serious, as will be known to anybody who has seen children with distended bellies and having retarded growth ${ }^{(6,7)}$. There are 1.3 billion helminthiasis infections globally( ${ }^{(8)}$. In most developing countries, helminthiasis incidence rates reached $90 \%{ }^{(9)}$. There are about 488 million human infections with $A$. lumbricoides occurred in Asia(10). In Bangladesh, the rate of infection is much higher due to over-population, climatic suitability of the parasites, poor socio-economic condition of the people, unplanned sewage system etc. Moreover, malnutrition and lack of awareness among people also aggravate the situation (7). The condition can be more fatal if left untreated ${ }^{(11-13)}$.

For successful control of ascariasis, efficient and reliable diagnosis is mandatory. However, Kato-Katz and Formol-ether concentration technique are two methods which are well appropriate to be used in the field for the diagnosis of Ascaris lumbricoides (AL) so far. Since poverty-strickon and unprivileged children are the prior victim of ascariasis, the present study will focus on the comparison between the two techniques to reveal the

*Author for correspondence: <hamida_khanum@yahoo.com>. ${ }^{1}$ International Centre for Diarrheal Disease Research, Bangladesh (ICDDR,B), Mohakhali, Dhaka, Bangladesh. 
easier, rapid, cheaper and more effective tool for the field diagnosis of AL. The motto of the experiment was to determine whether Formol-ether can be replaced by Kato-Katz technique with a moderate expenditure and reliable calculation. In 1954, Kato and Miura introduced a thick-smear technique for fecal examination ${ }^{(14)}$. Soon thereafter, Katz modified and adapted this technique for the use in field studies ${ }^{(15)}$. This method was adopted by WHO for both quantitative and qualitative diagnosis of Ascaris lumbricoides ${ }^{(16)}$.

\section{Materials and Methods}

A total of 205 children with symptoms of ascariasis were studied and their fecal samples were collected from the children of Bihari slum at Mirpur Section-11 during December, 2011 - March, 2012 and May - August, 2012. The children were between age range of 24 and 60 months and 94 and 159 months. Each stool sample was used for two different methods which were later compared. Microscopy was done for the confirmation of helminthes in the laboratory.

The Kato-Katz technique was modified according to Cheesbrough ${ }^{(17)}$. Small amount of fecal material was taken on a paper, a piece of nylon screen was pressed on top so that some of the feces were sieved through the screen and accumulated on the top. To count Ascaris eggs, the smear was examined in systematic manner and the number of eggs were multiplied by 24 to obtain the number of eggs per gram of feces (EPG).

The Formol-ether technique was performed according to the method described by Cheesbrough (1987)(18). The ample $(1 \mathrm{~g})$ was placed in a test tube and $5 \mathrm{ml}$ of normal saline was added and it was then centrifuged at $3000 \mathrm{rpm}$ for $3 \mathrm{~min}$ and supernatant was discarded. The process was repeated until the supernatant was clear. The sediment was examined using 10X objective with the condenser. The 40X objective was used to identify the small cysts and eggs. And the data generated was analyzed by the SPSS Software-15 for mean analysis, correlation and Chi square test.

\section{Results and Discussion}

For Ascaris lumbricoides the Kato-Katz (KK) showed mean of EPG (11130.89) higher than the mean EPG by Formol ether (1593.32). Mean for males (101) by KK was 11870.81 which were greater than mean EPG 1476.78 by FE. Again the mean by KK (10412.31) was higher than F E (1706.50) in female (Table 1). The mean difference showed better count by KK than FE which indicates better quantification of eggs of $A$. lumbricoides by K-K. Cross tabulation of $A$. lumbricoides by KK and FE showed positive counts in 149 samples which was about $72.68 \%$ of total samples (205). There were eight samples which showed positive counts for KK but negative for FE whereas, one sample was negative for KK but positive for FE. Among 205 samples (Table 2) KK was positive in 157 (76.58\%). FE was positive in 150 samples (73.2\%). 


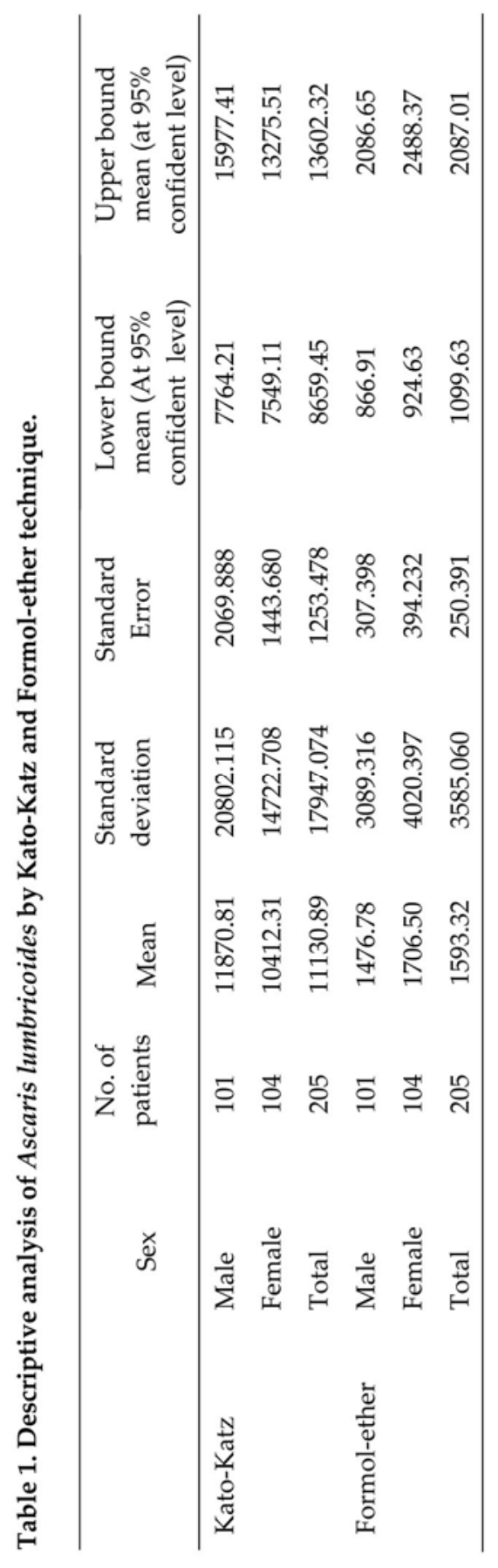


From the statistical analysis by SPSS, Pearson's correlation was significant between KK and FE. In Table 3, coefficient of correlation was significant at 0.01 level $\left(\mathrm{r}=0.453^{* *}\right)$ which clearly showed that EPG of A. lumbricoides counts were better derived by KK than FE.

Table 2. Cross tabulation of Ascaris lumbricoides by Kato-Katz and Formol ether technique

\begin{tabular}{lccc}
\hline Technique & $\begin{array}{c}\text { Formol ether } \\
\text { (Negative count) }\end{array}$ & $\begin{array}{c}\text { Formol ether } \\
\text { (Positive count) }\end{array}$ & Total \\
\hline Kato-Katz (Negative count) & 47 & 01 & 48 \\
Kato-Katz (Positive count) & 08 & 149 & 157 \\
Total & 55 & 150 & 205 \\
\hline
\end{tabular}

Table 3. Coefficient of correlation in case of Ascaris lumbricoides.

\begin{tabular}{llll}
\hline Technique & & Kato-Katz & Formol ether \\
\hline Kato Katz & Pearson correlation & 1 & $0.453^{* *}$ \\
& $\begin{array}{l}\text { Sig. (2-tailed) } \\
\text { no. of patients }\end{array}$ & 205 & 0.000 \\
& & $\begin{array}{l}\text { Formol ether } \\
\text { Pearson Correlation }\end{array}$ & 1 \\
& & $\begin{array}{l}\text { Sig. (2-tailed) } \\
\text { no. of patients }\end{array}$ & 205 \\
& &
\end{tabular}

${ }^{* *}$ Correlation was significant at 0.01 level

There were two batches of patients, one group was between the age of 24 - 60 months and the other was 94 - 159 months. Each group was analyzed separately according to sex and the mean EPG from each group was counted. The female patients ranging from the age limit between 24 - 63 and 94 - 159 were tested for their Ascaris egg counts separately by KK and FE technique. The mean EPG was significantly higher in KK in both the groups which again indicated the better counts by KK compared to FE (Fig. 1).

The 24 - 60 and 94 - 159 months age groups of male patients were also tested for $A$. lumbricoides egg counts by KK and FE methods. The mean EPG was always higher by KK except in age group 24-33 where the FE (mean EPG=582) counted higher eggs than KK (mean EPG=232). KK method in both groups which again indicates the better counts compared to FE (Fig. 2). The female patients ranging from the age limit between $24-60$ and 94 - 159 were tested for Ascaris egg counts separately by KK and FE techniques. The mean EPG was significantly higher in Kato-Katz.

Accurate and efficient diagnosis are the key for adequate patient management and guiding the design, implementation, and monitoring of community-based infectious disease control programs ${ }^{(19,20)}$. Both the $\mathrm{KK}$, a (semi) quantitative and FE are qualitative stool examination technique and generally recommended for diagnosis and evaluation of 
A. lumbricoides infection by the parasitologists. In the present experiment, the descriptive analysis of A. lumbricoides showed total mean EPG of 11130.89 by KK which was obviously higher than the mean analyzed for FE (1593.32). Cross tabulation of both KK and FE showed positive counts in 149 samples. There were eight samples which showed positive counts for KK but negative for FE. In cross-tabulation, both KK and FE showed positive counts in 149 samples. KK showed positive counts 10 in 157 samples whereas FE showed 150 positive counts out of 205.
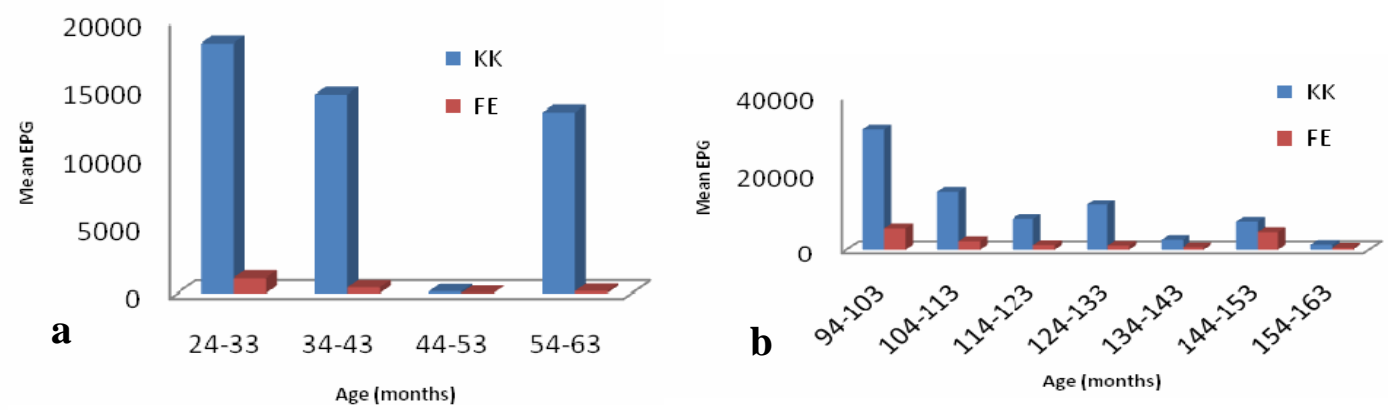

Fig. 1. (a,b) Composition of EPG of Ascaris lumbricoides in females of different age groups by KatoKatz and Formol ether methods.
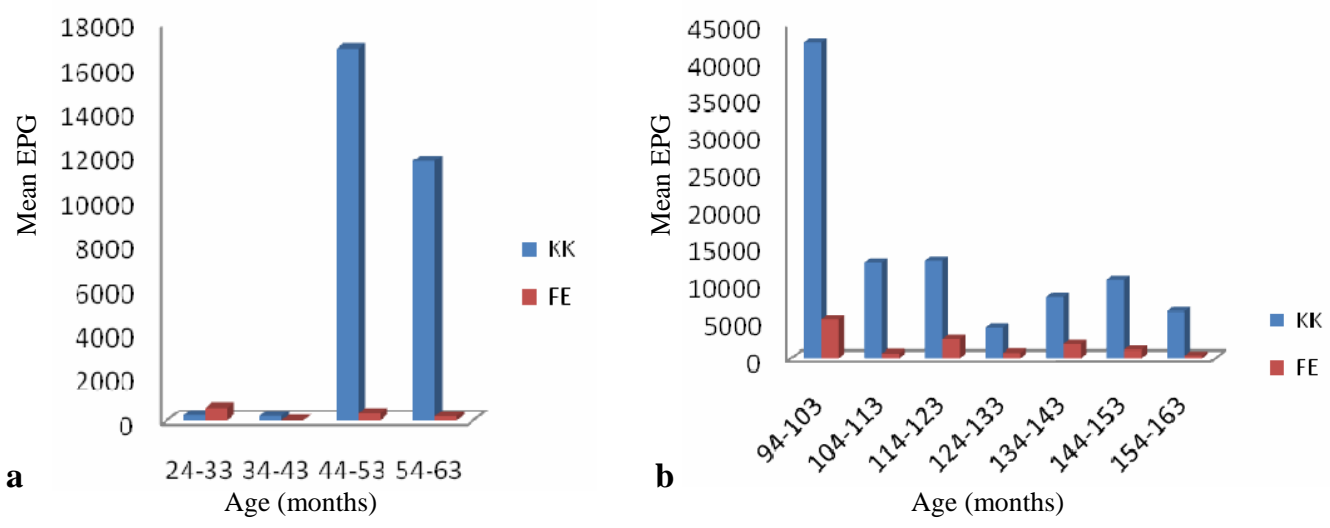

Fig. 2.(a,b). Comparison of EPG of Ascaris lumbricoides in males of age group 24 - 60 and 94 - 159 months by Kato-Katz and Formol ether methods.

The reason behind the high mean EPG by KK method might be due to the higher intensity of parasites ${ }^{(21)}$. Some other workers also reported that KK method was more sensitive for six groups of helminthes including A. lumbricoides(22-23). Prior work focusing on the blood fluke Schistosoma mansoni speculated that the multiple washing steps in the FECT could influence its sensitivity. Loss of eggs at washing steps might also explain the 
low EPGs observed in FE technique compared to KK method. As a consequence, $50 \%$ of eggs might have missed by FE method ${ }^{(23)}$. Some other workers woking with Opisthorchis viverrini also reported that the Kato-Katz method is considerably more sensitive than Formol-ether in detecting O. viverrini infection ${ }^{(24,25)}$. The calculation of EPG was based on the assumption that the density of a stool sample is equal to 1.0, as templates determine a defined volume, not a weight. Hence, any change in the consistency of stools might influence the results. A dry sample can have an egg count seven times as high as a wet sample obtained from the same subject(26).

In the present experiment, Pearson (2-tailed) Correlation was significant at the 0.01 level $\left(\mathrm{r}=0.453^{* *}\right)$. There was a positive relationship between these two methods. The quantitative analysis revealed that $\mathrm{KK}$ could be a better choice instead of FE for diagnosing ascariasis. Lovis et al.(24) reported that a single KK had significantly lower detection capacity than the FEC method in diagnosing hookworm which contradicts with the findings of this research(25). Because, hookworm has lower egg laying capacity, hookworm eggs are thin layered and easily disappear due to glycerin(26). But in case of $A$. lumbricoides, eggs are double layered and remain viable for long time. So KK thick smear is much more applicable for diagnosing ascariasis. Quantitative results of the KK technique remain valuable for estimating the intensity of infection in a population and to evaluate the evolution of Ascaris sp. endemicity. The KK method is more reliable than FECT for the diagnosis of $A$. lumbricoides. Therefore, the employment of FE technique as a confirmatory test in routine laboratory examination of stool and $\mathrm{KK}$ in epidemiological studies will significantly aid in accurate determination and management of parasitic infections in the community.

\section{References}

1. The World Health Organization 1998. Control of Tropical Diseases. World Health Organization, Geneva, Switzerland. pp.158.

2. Loreille $\mathrm{O}$ and $\mathrm{F}$ Bouchet 2003. Evolution of ascariasis in humans and pigs: A multidisciplinary approach, Memórias do Instituto Oswaldo Cruz Fundação Oswaldo Cruz, Fiocruz Mem Inst Oswaldo Cruz, Rio de Janeiro. 98: suppl-1: 39-46.

3. De Silva NH, L Guyatt and DAP Bundy 1997. Morbidity and mortality due to Ascaris induced intestinal obstruction. Trans. Royal Soc. Trop. Med. Hyg. 91: 31-36.

4. Ahmed FM, Mohiduzzaman and AA Jackson 1993. Vitamin A absorption in children with ascariasis. British J. Nut. 69: 817-25.

5. Braid JK 1986. Fatal human Ascaris following secondary massive infection. Amer. J. Trop. Med. Hyg. 35(2): 314-318

6. Denham DA 1984. Ascaris lumbricoides in English School Children. Trans. Royal Soc. Trop. Med. Hyg. 78: 566-567.

7. Alam MS and H Khanum 2005. Infection of Ascaris lumbricoides and Trichuris trichiura among the children of two slum areas in Dhaka city, Bangladesh. Bangladesh J. Zool. 33: 89-94. 
8. Jimenez B 2007. Alternative treatment for wastewater destined for agriculture use. Water Sci. Technol. 40: 355-362.

9. Bratton R and R Nesse 1993. Ascariasis: An infection to watch for in immigrants. Postgraduate Med. 93: 171-178.

10. Khanum H, S Chawdhury and ZJ Bhuiyan 1999. Infestation of three intestinal worms in children of three selected areas, Bangladesh. Pak. J. Zool. 31(4): 391-396.

11. Khanum H, N Islam and N Nahar 1999. Infestation of intestinal nematodes among the children in the residence of lower income group employees of Dhaka city. Bangladesh J. of Zool. 27(2): 177-184.

12. Rodriguez-Garcia AJ, J Belmares-Taboada and JF Hermandez-Sierra 2004. Ascaris lumbricoides caused risk factors for intestinal occlusion and subocclusion. Cirugia Cirujanos 72(1): 37-40.

13. Kato $\mathrm{K}$ and M Miura 1954. Comparative examinations. Jap. J. Parasitol. 3: 35.

14. Katz N, A Chavez and J Pellegrino 1972. A simple device for quantitative stool thick smear technique in Schistosoma mansoni. Revista do Instituto de Medicina Tropical de São Paulo 14: 397-400.

15. World Health Organization 1993. Faecal thick smear examination technique (Kato) for diagnosis of intestinal schistosomiasis and gastrointestinal helminth infections, World Health Organization. 83: 3.

16. Cheesbrough M 1991. Medical Laboratory Manual for Tropical Countries, Butterworth-Heinemann Ltd., Oxford, UK, 1: 178-197.

17. Cheesbrough M 1987. Medical Laboratory Manual for Tropical Countries. 2nd Edn, Butterworth and Co. Publishers, pp. 605.

18. Bergquist R, V Johansen and J Utzinger 2009. Diagnostic dilemmas in helminthology: What tools to use and when? Trends Parasitol. 25:151-156.

19. Peeling RW, PG Smith and PM Bossuyt 2006. A guide for diagnostic evaluations. Nature Reviews Microbiology 4: 2-6.

20. Knight WB, RA Hiatt, BL Cline and LS Richie 1976. A modification of the formol ether concentration technique for increased sensitivity in detection of Schistosoma mansoni eggs. Amer. J. Trop. Med. Hyg. 25: 87-97.

21. Hong ST, MH Choi, CH Kim, BS Chung and Z Ji 2003. The Kato-Katz method is reliable for diagnosis of Clonorchis sinensis infection. Diagnostic Microbiology and Infctious Diseases 47: 345-34.

22. Ebrahim A, AH El-Morshedy, E Omer, SE Daly and R Barakat 1997. Evaluation of the KatoKatz thick smear and formol ether sedimentation techniques for quantitative diagnosis of Schistosoma Mansoni. Amer. J. Trop. Med. Hyg. 57: 706-708.

23. Soukhathammavong P, P Odermatt, S Sayasone, Y Vonghachack, P Vounatsou, C Hatz, K Akkhavong and J Keiser 2011. Efficacy and safety of mefloquine, artesunate, mefloquineartesunate, tribendimidine, and praziquantel in patients with Opisthorchis viverrini: A randomised, exploratory, open label, phase 2 trial. Lancet Infect. Dis. 11: 110-118. 
24. Lovis L, TK Mak, K Phongluxa, P Soukhathammavong, Y Vonghachack, J Keiser, P Vounatsou, M Tanner, C Hatz, J Utzinger, P Odermatt and K Akkhavong 2012. Efficacy of praziquantel against Schistosoma sekongi and Opisthorchis viverrini: A randomized, singleblinded dose-comparison trial. PLOS Negleceted Tropical Diseases 6:17-26.

25. Teesdale $\mathrm{CH}, \mathrm{K}$ Fahringer and L Chitsulo 1985. Egg count variability and sensitivity of a thin smear technique for the diagnosis of Schistosoma mansoni. Trans. Royal Soc. Trop. Med. Hyg. 79: 369-373.

(Manuscript received on 11 March, 2014; revised on 6 July, 2014) 